\title{
ASSOCIAÇÃO DA FERRAMENTA DE TRIAGEM NUTRICIONAL STRONGKIDS COM PARÂMETROS ANTROPOMÉTRICOS EM CRIANÇAS
}

\author{
ASSOCIATION OF NUTRITIONAL SCREENING TOOL STRONGKIDS WITH \\ ANTHROPOMETRIC PARAMETERS IN CHILDREN
}

\section{ASOCIACIÓN DE LA HERRAMIENTA DE CLASIFICACIÓN NUTRICIONAL STRONGKIDS CON PARÁMETROS ANTROPOMÉTRICOS EN NIÑOS}

Liliane A. de Souza ${ }^{1}$, Marcella C.L. da Luz ${ }^{2}$, Isis S.C. Moura ${ }^{3}$, Tatiane Pontes Silva ${ }^{4}$

\begin{abstract}
RESUMO
Objetivos: descrever a prevalência de risco nutricional e verificar a associação da ferramenta de triagem nutricional STRONGkids com os índices antropométricos de peso para idade (P/I), estatura para idade (E/I) e IMC para idade (IMC/I) em pacientes pediátricos. Métodos: estudo transversal e analítico, realizado de abril a outubro de 2015, com 201 pacientes clínicos e cirúrgicos admitidos na enfermaria pediátrica de um hospital universitário no Nordeste brasileiro. A classificação do estado nutricional foi realizada por meio dos escores-z para os índices P/I, E/I e IMC/I e percentis para a circunferência braquial (CB). A ferramenta STRONGkids foi utilizada para determinar o risco de desnutrição. Resultados: a ferramenta STRONGkids revelou que 40,8\% dos pacientes apresentaram risco moderado e $4,5 \%$, alto risco para desnutrição. A STRONGkids teve associação significativa com a antropometria, uma vez que, quanto maior o risco nutricional, piores os índices antropométricos que avaliam desnutrição aguda e crônica. Conclusão: os resultados suportam o uso da ferramenta STRONGkids para identificar o risco nutricional em pacientes pediátricos internados, uma vez que a ferramenta mostrou associação com os parâmetros antropométricos de avaliação nutricional.
\end{abstract}

\section{Descritores: Nutrição da Criança; Pediatria; Desnutrição.}

\section{ABSTRACT}

Objectives: to describe the prevalence of nutritional risk and to verify the association of the nutritional screening tool STRONGkids with the anthropometric indices of weight to age (W/A), height to age (H/A) and BMI to age (BMI/A) in pediatric patients. Method: a cross-

\footnotetext{
${ }^{1}$ Nutricionista Residente. Universidade Federal de Pernambuco/UFPE. Recife (PE), Brasil.

2 Nutricionista. Universidade Federal de Pernambuco/UFPE. Recife (PE), Brasil.

3 Doutora. Universidade Federal de Pernambuco/UFPE. Recife (PE), Brasil.

${ }^{4}$ Graduanda em Nutrição. Centro Universitário Estácio do Recife. Recife (PE), Brasil.
}

Rev. Port. Saúde e Sociedade. 2019;4(1): 975 - 984. 
sectional and analytical study, carried out from April to October 2015, with 201 clinical and surgical patients admitted to the pediatric ward of a university hospital in the Brazilian Northeast. The classification of nutritional status was performed using the z-scores for the $\mathrm{W} / \mathrm{A}, \mathrm{H} / \mathrm{A}$ and BMI/A indices and percentile for arm circumference (AC). The STRONGkids tool was used to determine the risk of malnutrition. Results: the STRONGkids tool revealed that $40.8 \%$ of the patients presented moderate risk and $4.5 \%$, a high risk for malnutrition. STRONGkids had a significant association with anthropometry, since the higher the nutritional risk, the worse the anthropometric indices that evaluate acute and chronic malnutrition. Conclusion: the results support the use of the STRONGkids tool to identify nutritional risk in hospitalized pediatric patients, since the tool showed association with anthropometric nutritional assessment parameters.

\section{Descriptors: Child Nutrition; Pediatrics; Malnutrition.}

\section{RESUMEN}

Objetivos: describir la prevalencia de riesgo nutricional y verificar la asociación de la herramienta de clasificación nutricional STRONGkids con los índices antropométricos de peso para edad (P/E), estatura para edad (E/E) y el IMC para edad (IMC/E) en pacientes pediátricos. Métodos: estudio transversal y analítico, realizado de abril a octubre de 2015, con 201 pacientes clínicos y quirúrgicos admitidos en la enfermería pediátrica de un hospital universitario en el Nordeste brasileño. La clasificación del estado nutricional se realizó a través de las puntuaciones-z para los índices $\mathrm{P} / \mathrm{E}$, E/E e IMC/E y percentiles para la circunferencia braquial (CB). La herramienta STRONGkids se utilizó para determinar el riesgo de desnutrición. Resultados: la herramienta STRONGkids reveló que el $40,8 \%$ de los pacientes presentaron un riesgo moderado y un 4,5\%, alto riesgo para la desnutrición. La STRONGkids tuvo una asociación significativa con la antropometría, ya que, cuanto mayor es el riesgo nutricional, peores los índices antropométricos que evalúan la desnutrición aguda y crónica. Conclusión: los resultados soportan el uso de la herramienta STRONGkids para identificar el riesgo nutricional en pacientes pediátricos internados, una vez que la herramienta mostró asociación con los parámetros antropométricos de evaluación nutricional.

\section{Descriptores: Nutrición del Niño; Pediatría; Desnutrición.}

\section{INTRODUÇÃO}

A desnutrição é uma das doenças nutricionais mais importantes nos países em desenvolvimento devido à sua alta prevalência, aos prejuízos socioeconômicos, à relação com as taxas de mortalidade infantil e, também, tem sido associada ao aumento da morbimortalidade, do tempo de internação e, finalmente, a maiores custos com a hospitalização. ${ }^{1-4}$

Diversos estudos relatam uma alta prevalência da desnutrição na admissão hospitalar infantil e que $15 \%$ a $50 \%$ das crianças apresentam a piora do estado nutricional durante a internação, porém, ainda são escassas as pesquisas publicadas sobre este assunto. , $^{3,7}$

A triagem de risco nutricional é um método de baixo custo, não invasivo e facilmente aplicável à beira do leito. ${ }^{8}$ Apesar de a identificação precoce do risco

Rev. Port. Saúde e Sociedade. 2019;4(1): 975 - 984. 
nutricional por meio de um rastreio ser uma prática comum no atendimento clínico de adultos, na população infantil, tem sido dificultada pela falta de uma ferramenta de triagem nutricional adequada e validada no Brasil. ${ }^{9-11}$ Estes dados evidenciam a importância da identificação precoce do risco nutricional para uma intervenção adequada.

Entre as ferramentas de triagem, a STRONGkids mostrou-se a de maior aplicabilidade, pois é rápida e pode ser efetuada por qualquer profissional de saúde treinado, na admissão. ${ }^{12-13}$

A STRONGkids vem sendo escolhida na prática clínica em hospitais brasileiros por ser de fácil e rápida aplicação (em média, cinco minutos) e por apresentar resultados compatíveis com dados objetivos. ${ }^{14}$ Dessa forma, este estudo teve, como objetivos, descrever a prevalência do risco nutricional em pacientes pediátricos internados em um hospital universitário e verificar a associação da ferramenta de triagem nutricional STRONGkids aos índices antropométricos de P/I, E/I e IMC/I.

\section{MÉTODOS}

Trata-se de um estudo transversal e analítico, realizado com pacientes admitidos na enfermaria de pediatria no Hospital das Clínicas da Universidade Federal de Pernambuco (HC-UFPE), durante o período de abril a outubro de 2015. Foram incluídos pacientes de ambos os sexos, com idades entre um mês e 18 anos, com um tempo mínimo de 24 horas de internamento. Foram excluídos aqueles cujos responsáveis não soubessem informar os dados necessários para a ferramenta de triagem, pacientes que necessitavam de cuidados intensivos, portadores de necessidades especiais, pacientes que passaram por cirurgia abdominal recente, com doenças causadas por erros inatos do metabolismo, impossibilitados de realizar a avaliação antropométrica e com patologias, como as cardiopatias congênitas. O estudo foi aprovado pelo Comitê de Ética e Pesquisa do Centro de Ciências da Saúde da instituição, sob o CAAE 41041014.0.0000.5208/2015.

O instrumento para a triagem nutricional STRONGkids foi aplicado em até 48 horas após a admissão. O questionário consiste em quatro itens: avaliação nutricional subjetiva; doença de alto risco nutricional ou cirurgia de grande porte; ingestão e/ou perdas nutricionais e a perda ou ganho ponderal insuficiente nas

Rev. Port. Saúde e Sociedade. 2019;4(1): 975 - 984. 
últimas semanas ou meses. A cada item, foi atribuída uma pontuação de zero a dois pontos, com uma pontuação máxima total de cinco pontos, sendo zero pontos, um a três pontos e quatro a cinco pontos correspondentes a: baixo, médio e alto risco nutricional, respectivamente. ${ }^{13}$

Para a avaliação antropométrica, foram mensurados, no momento da admissão ou em até 48 horas após o internamento, o peso, a estatura e a circunferência braquial (CB), segundo as técnicas propostas pela OMS em 2008. O estado nutricional foi classificado por meio da curva de distribuição em escores-z para os índices estatura/altura para idade $(E / I)$, peso para idade $(P / I)$ e índice de massa corporal para idade (IMC/I). Os pontos de cortes utilizados na classificação do estado nutricional foram definidos de acordo com os critérios da OMS em 2006 e $2007^{15}$ e os valores de escores-z foram calculados com o auxílio do software WHO Anthro 2011, versão 3.2.2.

Foi mensurada a CB para se classificar a reserva muscular, apontando-se a baixa reserva muscular (desnutrição) para os percentis $<5$, dentro da média, entre os percentis 5 e 95, e alta reserva muscular para os percentis $>95 .{ }^{16}$

Os dados foram avaliados no SPSS, versão 13.0. As variáveis contínuas foram testadas quanto à normalidade da distribuição, pelo teste de Kolmogorov Smirnov. As variáveis com distribuição normal foram descritas sob a forma de médias e dos respectivos desvios-padrão. As variáveis com distribuição não gaussiana foram apresentadas sob a forma de medianas e dos respectivos intervalos interquartílicos. Para se verificar as associações entre as variáveis categóricas, foi aplicado o teste qui-quadrado. Para a comparação de médias, foi aplicada a análise de variância pelo ANOVA e post hoc de Tukey. O nível de significância adotado foi de $5 \%$.

\section{RESULTADOS}

Um total de 210 pacientes pediátricos foi coletado neste estudo, todavia, foram excluídos nove voluntários por não terem o seu peso e a estatura aferidos nas primeiras 48 horas de internamento hospitalar. Dessa forma, a população estudada foi composta por 201 pacientes, com a predominância do sexo masculino $(58,7 \%)$ e uma idade média de $82,8 \pm 60,5$ meses.

Rev. Port. Saúde e Sociedade. 2019;4(1): 975 - 984. 
A tabela 1 mostra que, entre as principais doenças que motivaram 0 internamento, se encontram doenças dos sistemas respiratório $(16,91 \%)$ e renal $(11,44 \%)$, bem como cirurgias pediátricas $(11,44 \%)$.

Em relação às condições socioeconômicas, a renda familiar mais prevalente foi de um a dois salários mínimos mensais, com $24,4 \%(n=49)$, seguida por $22,3 \%$ $(n=46)$ e $17,4 \%(n=35)$ de pacientes que recebiam um salário e menos que um salário mínimo, respectivamente. Quanto à escolaridade dos pais ou responsáveis, apenas 14,43\% $(n=29)$ terminaram a educação básica (oito anos), 30,85\% $(n=62)$ terminaram o Ensino Médio $(11$ anos) e 10,9\% $(n=22)$ frequentaram o Ensino Superior (15 anos).

A figura 1 demonstra o risco nutricional dos pacientes avaliado pela STRONGkids. Os pacientes que apresentaram o médio $(n=81)$ e alto risco $(n=10)$ tinham, como os principais motivos de internamento, as doenças do trato gastrointestinal $(6,97 \% ; n=14)$, as doenças renais $(5,97 \% ; n=12)$, as doenças respiratórias $(5,47 \% ; n=11)$, as doenças endócrinas $(5,47 \% ; n=11)$ e as cirurgias pediátricas $(4,97 \% ; n=10)$.

Em relação à antropometria, a média para o peso foi de $27,16 \pm 18,2 \mathrm{~kg}$. Para a estatura, foi de $116 \pm 33 \mathrm{~cm}$ e, para o IMC, a média foi de 17,86 $\pm 4,04$ $\mathrm{kg} / \mathrm{m}^{2}$.

A tabela 2 mostra que a prevalência de desnutrição moderada e/ou grave (<-2 escore-z) foi de 13,0\% ( $n=18), 10,9 \%(n=22)$ e 9,45\% $(n=19)$, para os índices $\mathrm{P} / \mathrm{I}, \mathrm{E} / \mathrm{I}$ e $\mathrm{IMC} / \mathrm{I}$, respectivamente. Já para a $\mathrm{CB}$, foi encontrada a desnutrição em $10,5 \%$ dos casos. Com relação à perda de peso observada no momento da admissão, 23,9\% $(n=48)$ apresentaram uma perda significativa de peso, com média de $2,85 \pm 2,72 \mathrm{~kg}$ por dia.

A relação entre o escore-z e os grupos de risco da STRONGkids está descrita na tabela 3. Observa-se que, quanto maior o risco nutricional, menor o escore-z para os índices antropométricos P/I $(p=0,00)$, E/I $(p=0,01)$ e IMC/I $(p=0,00)$.

Tabela 1. Descrição das características demográficas e diagnósticas de pacientes pediátricos internados.

\begin{tabular}{lccc}
\hline Variável & Categoria & n & \% \\
\hline Sexo & Masculino & 118 & 58,7 \\
& Feminino & 83 & 41,3 \\
Idade (meses) & $\leq 60$ & 90 & 44,8
\end{tabular}

Rev. Port. Saúde e Sociedade. 2019;4(1): 975 - 984. 
Diagnósticos

Cirurgias ortopédicas

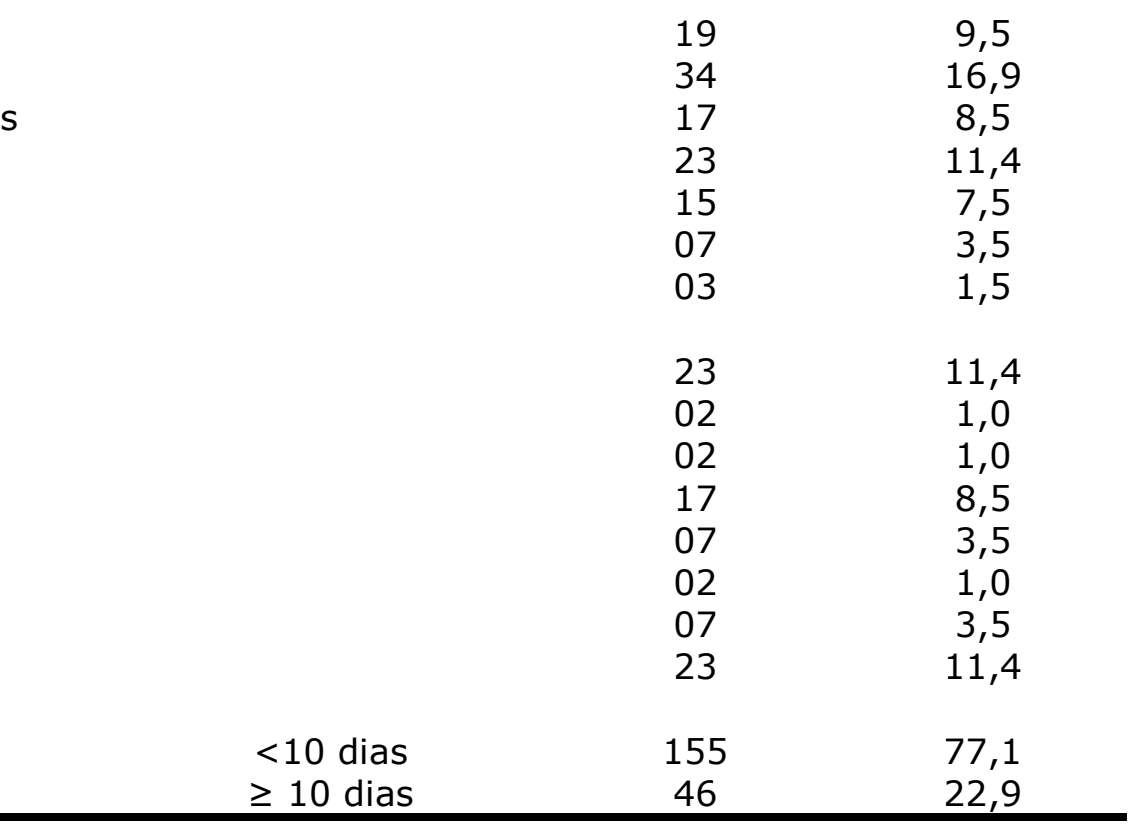

Doenças respiratórias

Doenças gastrointestinais

Doenças renais

Doenças endócrinas

Alergia alimentar

Cirurgias de

Otorrinolaringologia

Cirurgias pediátricas

Doenças urológicas

Doenças parasitárias

Doenças dermatológicas

Doenças reumáticas

Desnutrição

Doenças hematológicas

Outras doenças

Tempo de permanência

Figura 1. Risco nutricional de acordo com a ferramenta STRONGkids.

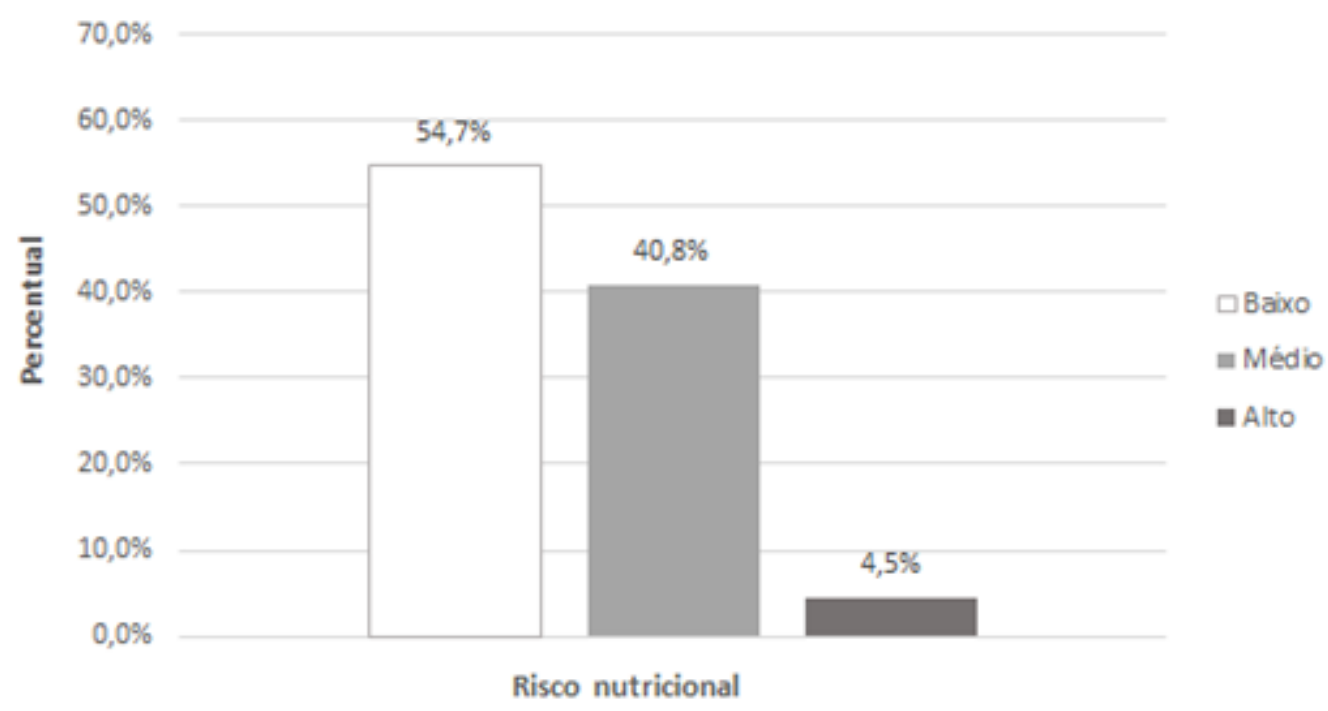

Tabela 2. Classificação do estado nutricional de pacientes pediátricos internados, segundo escore-z de PI, EI, IMC/idade e CB.

\begin{tabular}{lcc}
\hline \multicolumn{1}{c}{ Antropometria } & $\mathbf{n}$ & $\mathbf{\%}$ \\
\hline P/I & 138 & 3,6 \\
$\begin{array}{l}\text { Muito baixo peso para a } \\
\text { idade }(<Z-3)\end{array}$ & 05 & 9,4 \\
$\begin{array}{l}\text { Baixo peso para a idade } \\
(\geq Z-3 \text { e }<Z-2)\end{array}$ & 13 & 77,6 \\
$\begin{array}{l}\text { Peso adequado para a idade } \\
(\geq Z-2 \text { e }<Z+2)\end{array}$ & 107 & 9,4 \\
$\begin{array}{l}\text { Peso elevado para a idade } \\
(>Z+2)\end{array}$ & 13 &
\end{tabular}

Rev. Port. Saúde e Sociedade. 2019;4(1): 975 - 984. 
E/I

Muito baixa estatura para a idade $(<Z-3)$

Baixa estatura para a idade

( $\geq Z-3$ e $<Z-2$ )

Estatura adequada para a

idade ( $\geq Z$-2)

IMC/I

Magreza acentuada $(<Z-3)$

Magreza ( $\geq Z-3$ e $<Z-2$ )

Eutrofia ( $\geq Z-2$ e $\leq Z+1)$

Risco de sobrepeso /

sobrepeso ( $\geq Z+1$ e $\leq Z$

+3 )

Obesidade $(>Z+3)$

CB

Desnutrição $(<p 5)$

Eutrofia (p5-p95)

Excesso de peso (>p95)

89,0

201

Tabela 3. Relação entre o escore-z e os grupos de risco STRONGkids de pacientes pediátricos internados.

\begin{tabular}{lccccc}
\hline \multicolumn{7}{c}{ Classificação STRONGkids } \\
\hline & $\mathrm{n}$ & Risco baixo & Risco médio & Alto risco & $\mathrm{p}$ \\
P/I (Escore-z) & 138 & $0,73 \pm 1,33^{\mathrm{a}}$ & $-0,20 \pm 1,67^{\mathrm{b}}$ & $-2,10 \pm 1,69^{\mathrm{c}}$ & 0,00 \\
E/I (Escore-z) & 201 & $0,12 \pm 1,46^{\mathrm{a}}$ & $-0,42 \pm 1,70^{\mathrm{b}}$ & $-2,13 \pm 2,13^{\mathrm{c}}$ & 0,01 \\
IMC/I (Escore-z) & 201 & $0,90 \pm 1,50^{\mathrm{a}}$ & $-0,23 \pm 1,74^{\mathrm{b}}$ & $-1,41 \pm 1,25^{\mathrm{b}}$ & 0,00 \\
\hline
\end{tabular}

Letras diferentes indicam diferença significativa no teste pos-hoc (Tukey).

\section{DISCUSSÃO}

Há evidências de que a desnutrição leva a sérios prejuízos no crescimento, aumenta a morbimortalidade e pode, também, estar associada a deficits comportamentais e cognitivos em períodos mais tardios da infância. ${ }^{14,17} \mathrm{~A}$ desnutrição é comum entre as crianças hospitalizadas, mas é, muitas vezes, negligenciada ou tratada de forma inadequada. ${ }^{18}$ Portanto, a questão do risco nutricional em crianças e adolescentes hospitalizados requer a atenção imediata de nutricionistas e pediatras.

Durante os últimos anos, a incidência do risco nutricional em crianças hospitalizadas, avaliadas por ferramentas de triagem de risco nutricional, foi verificada em vários países. Quando comparada aos achados desta pesquisa, a maioria dos estudos descritos na literatura apresenta percentuais de alto risco nutricional superiores (4,5\%), como é o caso que analisaram 296 pacientes pediátricos em Paris e constataram que $44,3 \%$ das crianças foram classificadas

Rev. Port. Saúde e Sociedade. 2019;4(1): 975 - 984. 
com um alto risco nutricional, porém, com resultados semelhantes para o risco moderado, de 40,9\%.17 Usando STRONGkids, avaliaram um grupo de crianças hospitalizadas na Holanda e mostraram que $8 \%$ possuíam alto risco e 54\% estavam em moderado risco nutricional. ${ }^{13} \mathrm{Na}$ Romênia, com 271 pacientes internados por motivos clínicos, observaram 23,7\% de pacientes com alto risco nutricional. ${ }^{19} \mathrm{Na}$ China, com 1325 pacientes internados por motivos cirúrgicos ou clínicos, mostrou-se uma prevalência de $9,1 \%$ para alto risco nutricional. ${ }^{14} \mathrm{Na}$ Itália, com 144 pacientes admitidos por motivos clínicos, demonstraram 15\% de alto risco de desnutrição. ${ }^{20} \mathrm{Na}$ Turquia, um estudo com 494 pacientes pediátricos internados por motivos cirúrgicos, utilizando a ferramenta STRONGkids, apresentou resultados inferiores aos deste estudo, com somente 1,2\% dos pacientes sendo considerados de alto risco para a desnutrição. ${ }^{18}$

Apesar de este estudo ter sido desenvolvido no Nordeste do Brasil, uma das regiões menos desenvolvidas do país, com condições socioeconômicas desfavoráveis, bem como um baixo nível de escolaridade dos pais ou responsáveis pelas crianças pesquisadas, o percentual de alto risco nutricional encontrado, quando comparado ao da literatura, é considerado baixo. Uma das possíveis explicações para tal achado pode ser o motivo do internamento hospitalar, uma vez que a maioria das doenças não possui uma relação direta com o estado nutricional. Além disso, o hospital onde foi realizada a pesquisa não é considerado de referência para o internamento de pacientes pediátricos e não possui uma unidade de terapia intensiva. Dessa forma, restringe-se o internamento relativo a patologias mais graves que poderiam estar mais relacionadas ao risco nutricional.

Ao considerar a associação da ferramenta STRONGkids aos parâmetros antropométricos, foi observado que, quanto maior o risco nutricional, menor o escore-z para os índices antropométricos P/I, E/I e IMC/I. Resultados semelhantes verificaram que o aumento do risco de desnutrição foi significativamente associado a uma maior prevalência, tanto da desnutrição aguda quanto da crônica. ${ }^{18}$ Também demonstraram que a média do escore-z para PI, EI e IMCI foi significativamente menor nas crianças com alto risco nutricional, em relação àquelas com risco baixo ou moderado. ${ }^{13-14,21}$

Em um estudo de validação da ferramenta STRONGkids, na Holanda, em 2010, foi observado que a mesma foi aplicada com êxito em 98\% das crianças hospitalizadas em uma pesquisa multicêntrica, com 44 hospitais pediátricos. Neste estudo, a ferramenta previu e relacionou o alto risco aos escores-z negativos para

Rev. Port. Saúde e Sociedade. 2019;4(1): 975 - 984. 
os parâmetros antropométricos de avaliação nutricional em pediatria, com maior perda de peso, maiores taxas de infecções e tempo de hospitalização prolongado. ${ }^{13}$

$\mathrm{O}$ uso de qualquer ferramenta de triagem para identificar as crianças em risco de desnutrição só pode ser considerado eficaz e razoável se resultar na intervenção precoce e em melhores resultados clínicos. Estudos de maior escala e longitudinais em pacientes pediátricos hospitalizados parecem ser necessários para se investigar se a desnutrição vai se desenvolver ou não em pacientes classificados como de alto risco de desnutrição por meio da STRONGkids. Todavia, este estudo demonstrou a associação da ferramenta aos índices de avaliação do estado nutricional de pacientes pediátricos e que mais pesquisas devem ser realizadas para corroborar os achados. Além disso, estudos de correlação e validação devem ser desenvolvidos para se garantir a segurança do uso desta ferramenta para a população pediátrica hospitalizada.

\section{CONCLUSÃO}

Verificou-se que a ferramenta STRONGkids pode ser útil e viável para identificar crianças e adolescentes em risco de desnutrição em um hospital pediátrico do Nordeste brasileiro, uma vez que foi capaz de demonstrar a associação entre o risco nutricional e os índices antropométricos.

\section{REFERÊNCIAS}

1. Eufrásio REM. Avaliação nutricional subjetiva global em pediatria: Adaptação e análise de um questionário [monography]. Natal: UFRN; 2014.

2. Carvalho FC, Lopes CR, Vilela LC, Vieira MA, Rinaldi AEM, Crispim CA. Translation and cross-cultural adaptation of the Strongkids tool for screening of malnutrition risk in hospitalized children. Rev Paul Pediatr. 2013 June; 31(2):159-65. Doi: http://dx.doi.org/10.1590/S0103-05822013000200005

3. Hartman C, Shamir R, Helcht C, Koletzko B. Malnutrition screening tools for hospitalized children. Curr opin clin nutr metab care. 2012 May; 15(3):303-9. Doi: 10.1097/MCO.0b013e328352dcd4

4. Wonoputri N, Djais JTB, Rosalina I. Validity of nutritional screening tools for hospitalized children. Rev metab nutr. 2014; 38:1-6. Doi: http://dx.doi.org/10.1155/2014/143649

5. Joosten KFM, Hulst JM. Nutritional screening tools for hospitalized children: Methodological considerations. Clin nutr. 2014 Feb; 33(1):1-5. Doi: 10.1016/j.clnu.2013.08.002

Rev. Port. Saúde e Sociedade. 2019;4(1): 975 - 984. 
6. Joosten $\mathrm{KF}$, Zwart $\mathrm{H}$, Hop WC, Hulst JM. National malnutrition screening days in hospitalized children in the Netherlands. Arch dis child. 2010 Feb; 95:141-5. Doi: 10.1136/adc. 2008.157255

7. Mehta NM, Corkins MR, Lyman B, Malone A, Goday PS, Carney LN, et al. Defining pediatricmalnutrition: a paradigm shift toward etiology-related definitions. JPEN J parenter enteral nutr. 2013 July; 37(4):460-81. Doi: 10.1177/0148607113479972

8. Medeiros $A Q$, Pinto ICS, Silva CP. Avaliação nutricional. In: Vasconcelos MJOB, Barbosa JM, Pinto ICS, Lima TM, Araújo AFC. Nutrição Clínica: Obstetrícia e Pediatria. Rio de Janeiro: Medbook; 2011. p. 39-56.

9. Kondrup J, Allison SP, Elia M, Vellas B, Plauth M. ESPEN guidelines for nutrition screening 2002. Clin nutr. 2003; 22(4):415-21. Doi:10.1016/S02615614(03)00098-0

10. McCarthy H, Dixon M, Crabtree I, Eaton-Evans MJ, McNulty $H$. The development and evaluation of the Screening Tool for Assessment of Malnutrition in Paediatrics (STAMP) for use by healthcare staff. J hum nutr diet. 2012 Aug; 25(4):311-8. Doi: $10.1111 / \mathrm{j} .1365-277 X .2012 .01234 . \mathrm{X}$

11. White M, Lawson K, Ramsey R, Dennis N, Hutchinson Z, Soh XY, et al. A Simple Nutrition Screening Tool for Pediatric Inpatients. JPEN J parenter enteral nutr. 2016 Mar; 40(3):392-8. Doi: 10.1177/0148607114544321

12. Ling RE, Hedges $V$, Sullivan PB. Nutritional risk in hospitalised children: an assessment of two instruments. e-SPEN Eur e-J Clin Nutr Metab. 2011 June; 6(3):153-7. Doi: https://doi.org/10.1016/j.eclnm.2011.01.007

13. Hulst JM, Zwart H, Hop WC, Joosten KF. Dutch national survey to test the STRONG kids nutritional risk screening tool in hospitalized children. Clin Nutr. 2010 Feb; 29(1):106-11. Doi: 10.1016/j.clnu.2009.07.006

14. Cao J, Peng L, Li R, Chen Y, Li X, Mo B, Li X. Nutritional risk screening and its clinical significance in hospitalized children. Clin Nutr. 2014 June; 33:432-6. Doi: 10.1016/j.clnu.2013.06.009

15. World Health Organization. Child Growth Standards: Length/height-for-age, weight-for-age, weight-for-lenght, weight-for-height and body mass index-for-age. Methods and development [Internet]. Geneva: WHO;2006 [cited 2018 Aug 8]. Available from: https://www.who.int/childgrowth/standards/technical_report/en/

16. Frisancho AR. Anthropometric Standards for the Assessment of Growthand Nutritional Status. Michigan: The University of Michigan Press Ann Arbor;1990. DOI: $10.3998 /$ mpub. 12198

17. Sermet-Gaudelus I, Poisson-Salomon AS, Colomb V, Brusset MC, Mosser F, Berrier $\mathrm{F}$, et al. Simple pediatric nutritional risk score to identify children at risk of malnutrition. Am j clin nutr. 2000 July; 72(1):64-70. Doi: 10.1093/ajcn/72.1.64

18. Durakbaşa ÇU, Fettahoğlu S, Bayar A, Mutus M, Okur H. The Prevalence of Malnutrition and Effectiveness of STRONGkids Tool in the Identification of Malnutrition Risks among Pediatric Surgical Patients. Balkan Med J. 2014 Dec; 31(4):313-21. Doi: 10.5152/balkanmedj.2014.14374

19. Mărginean O, Pitea AM, Voidăzan S, Mărginean C. Prevalence and Assessment of Malnutrition Risk among Hospitalized Children in Romania. J Health Popul Nutr

Rev. Port. Saúde e Sociedade. 2019;4(1): 975 - 984. 
[Internet]. 2014 Mar [cited 2018 Aug 10]; 32(1): 97-102. Available from:https://www.ncbi.nlm.nih.gov/pubmed/24847598

20. Spagnuolo MI, Liguoro I, Chiatto F, Mambretti D, Guarino A. Application of a score system to evaluate the risk of malnutrition in a multiple hospital setting. Italian Journal of Pediatrics. 2013 Dec;39:81. Doi: 10.1186/1824-7288-39-81

21. Secker DJ, Jeejeebhoy KN. Subjective global nutritional assessment for children. Am J Clin Nutr. 2007 Apr; 85(4):1083-9. Doi: 10.1093/ajcn/85.4.1083

Rev. Port. Saúde e Sociedade. 2019;4(1): 975 - 984. 